\title{
Infant sleeping position and inspiratory pressures in the upper airways and oesophagus
}

\author{
Olav Skatvedt, Jens Grøgaard
}

\begin{abstract}
Inspiratory pressures were measured at three levels in the upper airways and in the oesophagus in different sleeping positions. Thirty one consecutive healthy infants were examined at the age of 1-3 days, 25 of these at 6 weeks, and 23 at 12 weeks. Oxygen saturation, an electrocardiogram, transthoracic impedance, nasal airflow, and inspiratory pressure in the epipharynx, oropharynx, hypopharynx, and in the intrathoracic part of the oesophagus were measured. There was no significant change in the heart rate, oxygen saturation, or respiratory rate in the four sleeping positions. The peak negative inspiratory pressures obtained from the oesophagus (PES) were significantly lower in the supine head straight compared with the supine head turned and the prone positions. At 6 weeks, the only significant difference was between the supine head straight and the prone positions. The PES is less negative at all ages in the supine head straight than in all other sleeping positions, but this does not reach statistical significance.

(Arch Dis Child 1994; 71: 138-140)
\end{abstract}

Studies have shown that sudden infant death syndrome (SIDS) occurs more often in the prone than in other sleeping positions. ${ }^{12}$ Previous studies have been mainly epidemiological ${ }^{3}$ and few have described physiological parameters in different sleeping positions. Hypotheses about possible pathological mechanisms have included oropharyngeal obstruction, ${ }^{4}$ rebreathing, ${ }^{5}$ compromised cerebral blood flow, ${ }^{6}$ and overheating. ${ }^{7}$

The incidence of SIDS has decreased in areas where the supine sleeping position has been advocated, but the rationale for this is still unclear. ${ }^{8}$ Obstructions of the upper airways during sleep have been suggested as a possible mechanism. ${ }^{9}$ Pharyngeal pressure changes during apnoeic episodes have implicated the pharynx as the site of obstruction. ${ }^{4}$

Multiple pressure transducers have been

Department of Otorhinolaryngology, Ullevaal, University Hospital, 0407 Oslo,

Norway

O Skatvedt

Department of

Pediatrics, Aker

University Hospital,

Oslo, Norway

J Grogaard

Correspondence to: Dr Skatvedt.

Accepted 22 April 1994
We hypothesised that the work of breathing differs in various sleeping positions. To sure transducers for simultaneous pressure test this hypothesis we used multiple pres- measurements at three different levels in the upper airways and in the oesophagus in various sleeping positions.

\section{Patients and methods}

Thirty one consecutive healthy newborn infants born at Aker University Hospital were examined. All parents had given their informed consent, the local medical ethical committee approved the protocol, and the equipment was certified by the official medical technical committee. The infants were first examined at the age of 1-3 days, and the examinations were repeated at 6 weeks and 3 months.

The tests included continuous measurement of oxygen saturation (Criticare), the electrocardiogram, and transthoracic impedance (SMART monitor, Healthdyne). An infant nasal thermistor (Healthdyne) was used for the detection of nasal airflow, but was not calibrated for flow volume.

Inspiratory pressures were measured in the epipharynx, oropharynx, hypopharynx, and in the intrathoracic part of the oesophagus (CAMLOG system, Camtech). The latter system has previously been described for use in adults for localising upper airway obstructions in obstructive sleep apnoea. ${ }^{1011}$ The sensitivity range of the pressure transducer is $\pm 100 \mathrm{~cm}$ $\mathrm{H}_{2} \mathrm{O}$. Rapid pressure changes of $10000 \mathrm{~Hz}$ can be detected, but the routine sampling frequency is only $5-10 \mathrm{~Hz}$.

The pressure transducers were located in a silicone tube (figure). The pressure transducer consists of a diaphragm covering a Wheatstone bridge. This bridge is part of an electrical circuit able to change the electrical resistance according to the tension of the bridge. When tension is applied to the system, the resistance of the Wheatstone bridge changes, resulting in a change in the signal voltage. This signal is calibrated, giving a numerical pressure value.

The four inspiratory pressures were obtained from pressure transducers in two different tubes (size $5 \mathrm{~F}$ : $1.6 \mathrm{~mm}$ in diameter) instrumented with three microtransducers in one and one microtransducer in the other (Camtech). A pH electrode was located at the end of tube 2 , but $\mathrm{pH}$ was not monitored in this study. The distance between each of the three more cranial transducers was $15 \mathrm{~mm}$, whereas the more rostral transducer was located in the oesophagus, $75 \mathrm{~mm}$ caudal to the hypopharyngeal transducer. The pressure transducers were calibrated by a two point calibration procedure before each test. Both tubes were inserted through one nostril.

The infants were fed 30 minutes before the study started to facilitate natural sleep. 


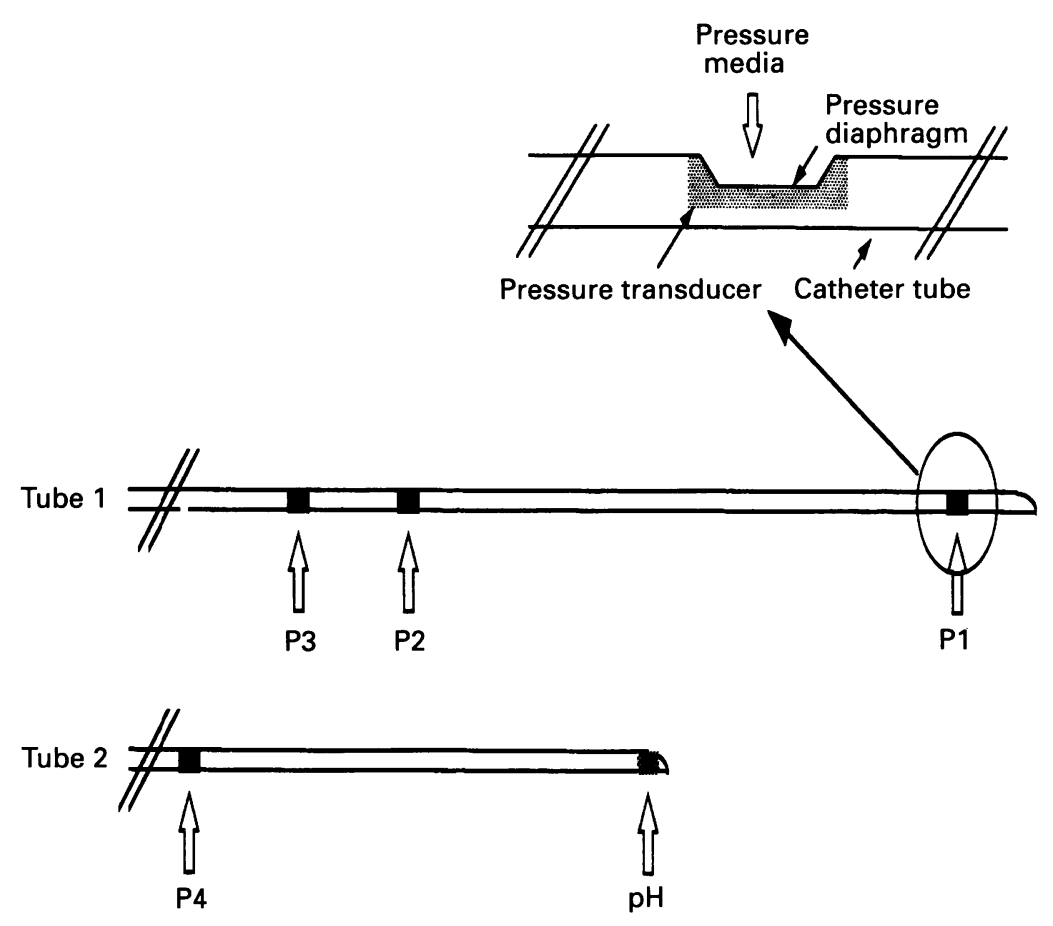

Two silicone tubes used: three pressure transducers (P1-3) in tube 1 and one pressure transducer (P4) and one pH sensor in tube 2. Each tube has a circumference of $5 F$ (diameter $1.6 \mathrm{~mm}$ ). Details of the pressure transducer are also shown.

Instrumentation was performed without any complications, and all infants slept calmly for at least five minutes in each sleeping position. The sleep stages were clinically judged according to Prechtl's staging. All results reported were obtained when the children were in quiet sleep.

At insertion of the tubes, an inert lubrication ointment without local anaesthetic effect was used. The infants were studied in the following sleeping positions: supine with the head turned, supine with the head straight, and prone. At the follow up examinations at 6 and 12 weeks of age, the protocol was extended to also include recordings in the lateral sleeping position (side).

All measurements were performed during quiet sleep. Inspiratory pressure was defined as the mean peak inspiratory pressure in 10 consecutive inspirations. Respiratory and heart rates were average rates calculated from two minutes of measurement, as was the oxygen saturation. All measurements in the same sleeping position were from the same time span.

Statistical calculations were performed by paired $t$ tests; $\mathrm{p}<0.05$ was considered statistically significant.

\section{Results}

Thirty one infants were studied during the first week of life; 25 were re-examined at 6 weeks and 23 at 12 weeks of age (table 1). Infants with any sign of airway infection were excluded at follow up.

Heart rate, respiratory rate, and oxygen saturation did not change significantly in different sleeping positions (table 2 ). The reduction in the respiratory rate with increasing age was significant (table 2) from newborn to 6 weeks, however, and from 6 weeks to 12 weeks.
Table 1 Characteristics of the children examined

\begin{tabular}{lccc}
\hline \multicolumn{4}{c}{ Age (weeks) } \\
\cline { 2 - 4 } & $\begin{array}{l}\text { Newborm } \\
(n=31)\end{array}$ & $\begin{array}{l}6 \\
(n=25)\end{array}$ & $\begin{array}{l}12 \\
(n=23)\end{array}$ \\
\hline Boys & 18 & 15 & 13 \\
Girls & 13 & 10 & 10 \\
Mean (SD) weight (g) & $3550(384)$ & $4956(604)$ & $6473(615)$ \\
\hline
\end{tabular}

Table 2 Mean (SD) respiratory rate, heart rate, and oxygen saturation in different sleeping positions

\begin{tabular}{lllll}
\hline $\begin{array}{l}\text { Age in } \\
\text { weeks }\end{array}$ & $\begin{array}{l}\text { Sleeping } \\
\text { position }\end{array}$ & $\begin{array}{l}\text { Respiratory } \\
\text { ratelmin }\end{array}$ & $\begin{array}{l}\text { Heart } \\
\text { ratelmin }\end{array}$ & $\begin{array}{l}\text { Oxygen } \\
\text { saturation (\%) }\end{array}$ \\
\hline $0(\mathrm{n}=31)$ & SHT & $53(11)$ & $124(15)$ & $95(3)$ \\
& SHS & $54(11)$ & $128(15)$ & $95(3)$ \\
& Prone & $53(12)$ & $131(15)$ & $95(4)$ \\
$6(\mathrm{n}=25)$ & SHT & $44(10)$ & $143(14)$ & $98(1)$ \\
& SHS & $43(9)$ & $141(11)$ & $98(1)$ \\
& Prone & $44(11)$ & $143(11)$ & $98(1)$ \\
& Side & $43(11)$ & $145(11)$ & $98(1)$ \\
$12(\mathrm{n}=23)$ & SHT & $33(11)$ & $130(5)$ & $97(1)$ \\
& SHS & $33(11)$ & $139(20) 98(1)$ & $98(1)$ \\
& Prone & $34(13)$ & $136(11)$ & $98(1)$ \\
& Side & $31(14)$ & $135(10)$ & $97(1)$ \\
& & & &
\end{tabular}

SHT = supine, head turned; SHS=supine, head straight; side $=$ lateral position.

The peak negative inspiratory oesophageal pressure (PES) in the supine head straight position was significantly lower than in the supine head turned $(p<0.03)$ and the prone positions $(p<0.001)$ at birth. At 6 weeks of age, the PES was significantly lower in the supine head straight than in the prone position $(p<0.02)$ (table 3). Overall, the PES is less negative at all ages in the supine head straight position than in all other sleeping positions, but none of the other comparisons reached statistical significance.

Hypopharyngeal, oropharyngeal, and epipharyngeal pressures showed no significant change in different sleeping positions at the same age. As for the PES, however, there is a tendency to reduced hypopharyngeal, oropharyngeal, and epipharyngeal pressures in the supine head straight position compared with all other sleeping positions.

The PES increased (increased negative values) with increasing age in the two supine positions. In the prone position the PES increased from newborn to 6 weeks of age, but decreased from 6 to 12 weeks of age.

Table 3 Mean (SD) peak inspiratory pressure ( $\left.\mathrm{cm} \mathrm{H}_{2} \mathrm{O}\right)$ at various locations in different sleeping positions

\begin{tabular}{|c|c|c|c|c|}
\hline \multirow[b]{2}{*}{ Location } & \multirow[b]{2}{*}{ Position ${ }^{\star}$} & \multicolumn{3}{|l|}{ Age (weeks) } \\
\hline & & $\begin{array}{l}\text { Newborn } \\
(n=31)\end{array}$ & $\begin{array}{l}6 \\
(n=25)\end{array}$ & $\begin{array}{l}12 \\
(n=23)\end{array}$ \\
\hline Oesophagus & $\begin{array}{l}\text { SHT } \\
\text { SHS } \\
\text { Prone } \\
\text { Side }\end{array}$ & $\begin{array}{l}5 \cdot 6(2 \cdot 0) \dagger \\
4 \cdot 8(1 \cdot 2) \\
6 \cdot 2(2 \cdot 3) \ddagger\end{array}$ & $\begin{array}{l}7 \cdot 4(2 \cdot 1) \\
6 \cdot 8(2 \cdot 0) \\
8 \cdot 4(2 \cdot 6) \$ \\
7 \cdot 7(2 \cdot 6)\end{array}$ & $\begin{array}{l}8 \cdot 1(3 \cdot 8) \\
7 \cdot 5(2 \cdot 7) \\
7 \cdot 8(2 \cdot 5) \\
7 \cdot 6(3 \cdot 2)\end{array}$ \\
\hline Hypopharynx & $\begin{array}{l}\text { SHT } \\
\text { SHS } \\
\text { Prone } \\
\text { Side }\end{array}$ & $\begin{array}{l}1.5(2 \cdot 1) \\
1.0(1.3) \\
1.3(1.6)\end{array}$ & $\begin{array}{l}1.6(1.9) \\
1.3(2.3) \\
1 \cdot 1(2.4) \\
1.6(1.9)\end{array}$ & $\begin{array}{l}0.9(2.4) \\
0.4(1.0) \\
0.8(1.5) \\
0.5(3.2)\end{array}$ \\
\hline Oropharynx & $\begin{array}{l}\text { SHT } \\
\text { SHS } \\
\text { Prone } \\
\text { Side }\end{array}$ & $\begin{array}{l}1.0(1 \cdot 1) \\
0.5(0.8) \\
1.9(2.4)\end{array}$ & $\begin{array}{l}1.0(1.4) \\
1.1(2 \cdot 1) \\
0.9(1.7) \\
0.9(1.5)\end{array}$ & $\begin{array}{l}1.6(2.3) \\
1.8(2.1) \\
2.0(1.9) \\
2.4(2.9)\end{array}$ \\
\hline Epipharynx & $\begin{array}{l}\text { SHT } \\
\text { SHS } \\
\text { Prone } \\
\text { Side }\end{array}$ & $\begin{array}{l}0 \cdot 3(1 \cdot 1) \\
0 \cdot 3(1 \cdot 3) \\
1 \cdot 3(2 \cdot 3)\end{array}$ & $\begin{array}{l}0.5(1.5) \\
0.6(2.1) \\
1.5(2.9) \\
0.6(3.1)\end{array}$ & $\begin{array}{l}2.4(3.1) \\
1.9(2.8) \\
3.3(2.8) \\
2.5(3.4)\end{array}$ \\
\hline
\end{tabular}

*SHT=supine, head turned; SHS=supine, head straight; side=lateral position

†Difference SHS/SHT, newborn: $p<0.03$.

$\neq$ Difference SHS/prone, newborn: $p<0.001$.

@Difference SHS/prone, 6 weeks: $\mathrm{p}<0.02$. 
The oropharyngeal and epipharyngeal pressures also showed a tendency towards increasingly negative values with age. In the hypopharynx, however, the tendency was for a reduction in hypopharyngeal pressure at 12 weeks compared with the two younger age groups.

\section{Discussion}

This study has focused on measurements of upper airway pressures in different sleeping positions in normal healthy infants. The oesophageal pressure was higher in the prone position than in the supine position at birth. This change in transpulmonary pressure indicates a difference in the work of breathing in different sleeping positions. Negative oesophageal pressure was still significantly higher in the prone position at 6 weeks of age, but not at 3 months of age. This is probably due to changes in lung mechanics rather than airway obstruction, as pressure measurements in the hypopharynx, oropharynx, and epipharynx showed no significant difference with positional change. These changes are minor, however, as other physiological parameters such as heart rate, respiratory rate, and oxygen saturation did not reveal significant changes.

Other investigators have reported better oxygenation in the prone than in the supine position in the newborn period. ${ }^{12}$ This could not be verified in this study as all infants showed normal transcutaneous oxygen saturation throughout the study. Several of the previous studies have included preterm infants and infants with respiratory distress syndrome, which could explain the difference in results.

Newborn infants have approximately one third of the total airway resistance located in the nose. ${ }^{13}$ We would therefore expect that insertion of the pressure transducer tubes through the nose would increase nasal resistance and thereby change the respiratory pattern. The baseline values for respiratory rate, heart rate, or oxygen saturation did not change after insertion of the nasopharyngeal tubes. The baseline values for nasal airflow did not differ before and after insertion of the tubes, although measurement by thermistor is not sensitive to minor flow changes.

Measurements of the upper airway pressures confirmed that tube insertion did not cause a major increase in upper airway resistance as pressure fluctuations during tidal breathing did not increase significantly before and after the second tube was inserted.

Research has shown that newborn infants can obtain $70 \%$ of their tidal volume by mouth breathing. ${ }^{14}$ Oral airflow was not measured in this study, but is a possible explanation for the unchanged respiratory pattern after nasopharyngeal tube insertion.
To validate the equipment used, we recorded the PES in two infants using a standard oesophageal balloon and the microtransducer. The pressure recordings were obtained at the same time and from the same place in the oesophagus. We found that the pressure and the pressure changes during each respiratory movement were identical.

Artificial material such as the pressure transducer tubes used could potentially influence the normal upper airway patency and normal upper airway activity in newborn infants. The silicone tubes used are soft at body temperature, however. In addition, the tubes are small and are identical in diameter to regular infant feeding tubes. Subject activities such as oral feeding, sucking, and swallowing were performed during the study without any signs of a lack of distress or coordination. Newborn infants with periodic breathing may develop complete obstructions in the pharynx, ${ }^{14}$ but whether upper airway patency was increased due to the tubes cannot be excluded because of the short duration of each study period. A sleep study lasting all night is necessary to include all activity states and to evaluate possible intermittent airway obstructions.

The authors thank B Gjersøe of Camtech for technical help and valuable discussions during the development of the apparatus, and I W S Mair for comments on the final version of the manuscript. The equipment was financed by a grant from the Norwegian SIDS Society and the Norwegian Research the Nor

First presented at the annual meeting of the Norwegian Society of Perinatal Medicine, Bodø, Norway, November 1991.

1 Engelberts AC, de Jonge GA. Choice of sleeping position for infants: possible association with cot death. Arch Dis Child 1990; 65: 462-7.

2 Dwyer T, Ponsby A-LB, Newman NM, Gibbons LE. Prospective cohort study of prone sleeping position and sudden infant death syndrome Lancet 1991 ; 337: 1244-7.

3 American Academy of Pediatrics. AAP task force on infant positioning and SIDS. Pediatrics 1992; 89: 1122-6.

4 Mathew OP, Roberts JL, Thach BT. Pharyngeal airway obstruction in preterm infants during mixed and obstrucobstruction in preterm infants during mix

$5 \mathrm{Kemp}$ JS, Thach BT. Sudden death in infants sleeping on polystyrene-filled cushions. N Engl f Med 1991; 324: 1858-64.

6 Saternus KS, Koebke J, von-Tamaska L. Neck extension as a cause of ISDS. Forensic Sci Int 1986; 31: 167-74.

7. Ponsonby AL, Dwyer T, Gibbons LE, Cochrane JA, Jones ME, McCall JM. Thermal environment and sudden infant death syndrome. Case control study. BMF 1992;

Engelberts A. Cot death in the Netherlands; an epidemiological study. [MD thesis.] Amsterdam: VK University

9 Tomkin SL, Stuart JH, Witberg S. Obstruction of the upper airway as a mechanism of sudden infant death. Sleep 1980; 33: $375-82$

10 Skatvedt $O$. Continuous pressure measurements in the pharynx and esophagus during sleep in patients with obstructive sleep apnea syndrome. Laryngoscope 1992; 102: $1275-80$.

11 Skatvedt O. Localization of site of obstruction in snorers and patients with obstructive sleep apnea syndrome: a comparison of fiberoptic nasopharyngoscopy and pressure measurements. Acta Otolaryngol (Stockh) 1993; 113: 206-9.

12 Martin RJ, Herrell N, Rubin D, Fanaroff A. Effects of supine and prone positioning on arterial oxygen tension in the preterm infant. Pediatrics 1979; 63: 528-31.

13 Lacourt G, Polgar G. Interaction between nasal and pulmonary resistance in newborn infants. $f$ Appl Phyiol 1971; 30: 870.

14 Miller MJ, Martin RJ, Carlo WA, et al. Oral breathing in newborn infants. $\mathcal{F}$ Pediatr 1985; 107: 465. 